Pacific Journal of Mathematic 


\section{STOLZ ANGLE CONVERGENCE IN METRIC SPACES}

\section{E. SNYDER}

A function $f$ defined on the real line is said to be a Stolz angle limit function if there is a function $\phi$ defined on the upper half-plane with property that at each point $(x, 0)$ there is a Stolz angle such that the boundary limit of $\phi$ relative to the Stolz angle exists and is equal to $f(x)$. In this paper the notion of Stolz angle convergence is extended for functions defined on metric spaces.

2. Definitions and notation. Let $(X, \rho)$ be a metric space and let $R^{+}$denote the positive real nuubers. A set in $X \times R^{+}$of the form

$$
\left\{(y, r) \in X \times R^{+}: \rho(x, y) \leqq a \cdot r\right\},
$$

where $a$ is some positive real number and $x$ is a point in $X$, is said to be a Stolz cone with vertex $x$. We will denote such a set by $C(x, a)$.

If $(X, \rho)$ is the real line with the usual metric, then a Stolz cone is a Stolz angle in the upper halfplane which has its vertex on the $x$-axis and which is symmetric about the line $x=c$ if $(c, 0)$ is its vertex.

Let $f: X \rightarrow R$. Then $\omega(x, f)$ denotes the oscillation of $f$ at $x$, for $x$ in $X$.

If $(X, \rho)$ is a metric space, we metrize $X \times R^{+}$with the metric $\rho$ ' defined by

$$
\rho^{\prime}((x, r),(y, s))=\max \{\rho(x, y),|r-s|\} \text {. }
$$

3. Continuous extensions. In the first theorem it is shown that a function $f$ in the first Baire class defined on a compact metric pace $X$ can be extended to a continuous function $\Phi$ on $X \times R^{+}$such that $f$ is the "nontangential" boundary limit of $\Phi$.

THEOREM 1. If $X$ is a compact metric space and if $f: X \rightarrow R$ is in the first Baire class, then there is a continuous function $\varphi: X \times R^{+} \rightarrow R$ such that for each $x \in X$,

$$
\lim \varphi(\mathrm{u}, r)=f(x) \quad \text { as }(u, r) \rightarrow(x, 0)
$$

relative to any Stolz cone $C(x, a)$.

Proof. Let $\left\{f_{n}\right\}$ be a sequence of continuous real-valued functions on $X$ such that $f_{n}(x) \rightarrow f(x)$ for each $x$ in $X$. Define $H: X \times(0,1] \rightarrow R$ 
as follows:

$$
\text { let } H\left(x, \frac{1}{n}\right)=f_{n}(x) \quad \text { for } n=1,2, \cdots \text {, }
$$

and extend linearly between $(x, 1 / n)$ and $(x, 1 / n+1)$. Clearly $H$ is continuous on $X \times[0,1]$, and $\lim H(x, r)=f(x)$ as $r \rightarrow 0^{+}$for each $x$ in $X$.

For each $n=1,2, \cdots$ and $\varepsilon>0$, let $\delta_{n}(\varepsilon)$ be a positive number such that $\rho(x, y)<\delta_{n}(\varepsilon)$ implies $\left|f_{n}(x)-f_{n}(y)\right|<\varepsilon$ for all $x$ and $y$ in $X$. We let $\zeta_{1}=\min \left\{\delta_{1}(1), \delta_{2}(1), 1\right\}$ and inductively define

$$
\zeta_{n}=\min \left\{\delta_{n}\left(\frac{1}{n}\right), \delta_{n+1}\left(\frac{1}{n}\right), \frac{\zeta_{n-1}}{2}\right\} \text {. }
$$

Then $\left\{\zeta_{n}\right\}$ is a decreasing sequence with limit zero. We define a function $\tau: R^{+} \rightarrow(0,1]$ by setting $\tau(r)=1$ if $r \geqq \zeta_{1}$, and $\tau(r)=1 / k$ if $r=\zeta_{k}$ and by extending linearly for $r$ between $\zeta_{k+1}$ and $\zeta_{k}$. This function $\tau$ is continuous. Also, the function $\Phi: X \times R^{+} \rightarrow R$ defined by

$$
\Phi(x, r)=H(x, \tau(r))
$$

is continuous.

We will verify that for each $x \in X, \lim \Phi(u, r)=f(x)$ as $(u, r) \rightarrow(x, 0)$ relative to $C(x, 1)$. To this end let $x_{0}$ be a fixed point in $X$, and suppose that $\left\{\left(x_{n}, r_{n}\right)\right\}$ is an arbitrary sequence of points in $C\left(x_{0}, 1\right)$ such that $\lim _{n \rightarrow \infty} r_{n}=0$ and hence $\lim _{n \rightarrow \infty} x_{n}=x_{0}$. Let $\varepsilon$ be an arbitrary positive number. There is a positive integer $K$ such that $1 / K<\varepsilon / 2$, and there is a positive integer $N_{1}$ such that $n>N_{1}$ implies $r_{n} \leqq \zeta_{K}$. Thus $\tau\left(r_{n}\right) \leqq 1 / K$ for $n>N_{1}$. Then from the definitions of $\left\{\zeta_{n}\right\}$ and the function $H$ and from the fact that $\rho\left(x_{0}, x_{n}\right) \leqq r_{n}$, it follows that

$$
\left|H\left(x_{n}, \tau\left(r_{n}\right)\right)-H\left(x_{0}, \tau\left(r_{n}\right)\right)\right| \leqq \frac{1}{K}<\frac{\varepsilon}{2}
$$

for $n>N_{1}$.

Since $\lim _{n \rightarrow \infty} \tau\left(r_{n}\right)=0$, it follows from the definition of $H$ that

$$
\lim _{n \rightarrow \infty} H\left(x_{0}, \tau\left(r_{n}\right)\right)=f\left(x_{0}\right) .
$$

Hence there is a positive integer $N_{2}$ such that $n>N_{2}$ implies

$$
\left|H\left(x_{0}, \tau\left(r_{n}\right)\right)-f\left(x_{0}\right)\right|<\frac{\varepsilon}{2} .
$$

Let $N=\max \left(N_{1}, N_{2}\right)$, and let $n>N$. Then, as a consequence of (1) and (2), we have 


$$
\left|\Phi\left(x_{n}, r_{n}\right)-f\left(x_{0}\right)\right|=\left|H\left(x_{n}, \tau\left(r_{n}\right)\right)-f\left(x_{0}\right)\right|<\varepsilon .
$$

Therefore,

$$
\lim _{n \rightarrow \infty} \Phi\left(x_{n}, r_{n}\right)=f\left(x_{0}\right) .
$$

The function $\Phi$ converges for the Stolz cones of the form $C(x, 1)$. We compose $\Phi$ with the homeomorphism $T: X \times R^{+} \rightarrow X \times R^{+}$which is defined by $T(x, r)=(x, \sqrt{r})$, and we let $\varphi$ denote the resulting function on $X \times R^{+}$, i.e., $\varphi(y, r)=\Phi(y, \sqrt{r})$.

Let $x_{0}$ be a fixed point in $X$ and let $C\left(x_{0}, a\right)$ be any Stolz cone with vertex $x_{0}$. Suppose $\left\{\left(x_{n}, r_{n}\right)\right\}$ is a sequence of points in $C\left(x_{0}, a\right)$ with $\left(x_{0}, 0\right)$ as limit. By definition of $C\left(x_{0}, a\right)$, we have $\rho\left(x_{0}, x_{n}\right) \leqq a \cdot r_{n}$. Also, for $r_{n} \leqq 1 / a^{2}$, we have $a \cdot r_{n} \leqq \sqrt{r_{n}}$. Consequently, $T\left(x_{n}, r_{n}\right)$ is in $C\left(x_{0}, 1\right)$ for $r_{n} \leqq 1 / a^{2}$, and $T\left(x_{n}, r_{n}\right) \rightarrow\left(x_{0}, 0\right)$. Therefore,

$$
\lim _{n \rightarrow \infty} \varphi\left(x_{n}, r_{n}\right)=\lim _{n \rightarrow \infty} \Phi\left(T\left(x_{n}, r_{n}\right)\right)=f\left(x_{0}\right),
$$

which concludes the proof.

It is interesting to note that when $X$ is a compact interval in $R^{n}$, the results obtained above are more general then Theorem 6 in [5]. Here it is shown that every Baire class one function $f$ of $n$ real variables is a boundary limit of a continuous function of $(n+1)$ real variables, whereas in [5] $f$ is shown to be a boundary limit of a continuous function of $2 n$ variables.

From the proof of Theorem 1, we see that the boundary limit of $\phi$ exists relative to the set

$$
T^{-1}[C(x, 1)] \text { for each } x \in X .
$$

This leads us to the following definition and corollary.

Definition. Let $g:[0, \infty) \rightarrow[0, \infty)$ be a homeomorphism. A set in $X \times R^{+}$of the form

$$
\left\{(y, r) \in X \times R^{+}: \rho(x, y) \leqq g(r)\right\}
$$

is called a generalized Stolz cone with vertex $x \in X$, and this set is denoted by $G(x, g)$.

Corollary. Let $X$ be a compact metric space and $f: X \rightarrow R$ a function in the first Baire class. Suppose that $g:[0, \infty) \rightarrow[0, \infty)$ is a homeomorphism. Then there is a continuous function

$$
\varphi: X \times R^{+} \rightarrow R
$$

with the property that for each $x \in X, \lim \varphi(u, r)=f(x)$ as $(u, r) \rightarrow(x, 0)$ 
relative to $G(x, g)$.

Proof. Define $T: X \times R^{+} \rightarrow X \times R^{+} \quad$ by $\quad T(x, r)=\left(x, g^{-1}(r)\right)$. Clearly $T$ is a homeomorphism. Also it is easily verified that $T(C(x, 1))=$ $G(x, g)$. Thus it suffices for the proof to define $\varphi=\Phi \circ T^{-1}$, where $\Phi$ is the continuous function constructed in the proof of Theorem 1.

4. Boundary functions. A function $f$ defined on a metric space $X$ is said to be a boundary function for the function $\Phi$ defined on $X \times R^{+}$if for each $x \in X, \lim \Phi(u, r)=f(x)$ as $(u, r)$ approaches $(x, 0)$ relative to some set in $X \times R^{+}$, which has $(x, 0)$ as a boundary point in $X \times[0, \infty)$.

In these considerations we concern ourselves only with metric spaces which are $F_{I I}$ spaces. An $F_{I I}$ metric space is defined by Hausdorff ([1], p. 165) to be a metric space for which every closed subset is second category in itself. It was proved by Hurewicz [2] that a space is an $F_{I I}$ space if and only if every nonempty perfect set is uncountable. Also, it is clear that any complete space is an $F_{I I}$ space.

THeOREm 2. Let $X$ be a $F_{I I}$ metric space. Suppose there is a function $\Phi: X \times R^{+} \rightarrow R$ with the property that for each $x \in X$ there is a homeomorphism $h_{x}:[0, \infty) \rightarrow[0, \infty)$ such that $\lim \Phi(u, r)$ exists as $(u, r)$ approaches $(x, 0)$ relative to the generalized Stolz cone $G\left(x, h_{x}\right)$. Then the boundary function $f$ determined by this family of generalized Stolz cones is in the first Baire class.

Proof. For the proof we will verify that for any nonempty perfect set $P, f \mid P$ has a point of continuity. Thus, by Baire's theorem (see [1], p. 288 or [3], p. 301, 2nd ed.), $f$ is in the first Baire class.

Suppose that $P \subset X$ is a nonempty perfect set and that $f \mid P$ has no point of continuity. Let

$$
D_{n}=\left\{x \in P: \omega(f \mid p, x) \geqq \frac{1}{n}\right\} .
$$

Since $f \mid P$ has no points of continuity, it follows that $P=\bigcup_{n=1}^{\infty} D_{n}$. Since $X$ is an $F_{I I}$ space, $P$ is of the second category in itself, so there exists an integer $n_{0}$ and an open sphere $\sigma$ such that $D_{n_{0}}$ is dense in $\sigma \cap P$ and $\sigma \cap P$ is nonempty. However, each $D_{n}$ is closed as the oscillation function is upper semi-continuous. Thus $D_{n_{0}} \supset \sigma \cap P$. Let $Q=I(P \cap \sigma)$. Then $Q$ is also a perfect set and $\omega(f \mid Q, x) \geqq 1 / n_{0}$ for $x \in \sigma \cap P$. 
Let

$$
G\left(h_{x}, x\right)_{n}=\left\{(y, r) \in G\left(h_{x}, x\right): r \leqq \frac{1}{n}\right\}
$$

and let

$$
A_{n}=\left\{x \in Q: q \in G\left(h_{x}, x\right)_{n} \Rightarrow \mid f(x)-\Phi(q)<1 / K\right\},
$$

where $K=16 n_{0}$. Since the Stolz limit of $\Phi$ equals $f(x)$ at each point, it follows that $Q=\bigcup_{n=1} A_{n}$. $Q$ being of the second category in itself implies that there exists an integer $k$ and an open shere $\tau$ such that $Q \cap \tau$ is nonempty and $A_{k}$ is dense in $Q \cap \tau$.

Let $z \in A_{k} \cap \tau \cap \sigma$ be fixed. Then $G\left(h_{z}, z\right)_{k} \cap G\left(h_{x}, x\right)_{k} \neq \phi$ for any $x$ for which $\rho(z, x) \leqq h_{z}(1 / k)$. In particular the point $(x, 1 / k)$ is in $G\left(h_{x}, x\right)_{k}$ and also is in $G\left(h_{z}, z\right)$ since $\rho(z, x) \leqq h_{z}(1 / k)$. Let $\sigma^{\prime}$ be the open sphere with center $z$ and radius $h_{z}(1 / k)$, and let $V=\sigma \cap \tau \cap \sigma^{\prime}$.

For each $x \in Q$, let $n(x)$ denote the smallest positive integer $n$ for which $x \in A_{n}$. Suppose that $\left\{x_{2}\right\}$ is any sequence of points in $P$ with $z$ as limit. Then there is an integer $N$ such that $i>N$ implies $x_{i}$ is in $V$. Let $i>N$ be fixed, and set $m=\max \left\{n\left(x_{i}\right), k\right\}$. Since $A_{k}$ is dense in $V \cap Q$, there is a $y \in A_{k} \cap V$ such that $\rho\left(x_{i}, y\right) \leqq h_{x_{i}}(1 / m)$. This implies that the point $\xi=(y, 1 / m)$ is in $G\left(h_{y}, y\right)_{m}$ and also in $G\left(h_{x_{i}}, x_{i}\right)_{m}$. Moreover, it is true that $\eta=(y, 1 / k)$ is in

$$
G\left(h_{y}, y\right)_{k} \cap G\left(h_{z}, z\right)_{k},
$$

for $y$ is in $A_{k} \cap V$. Thus it follows that

$$
\begin{aligned}
\left|f(z)-f\left(x_{i}\right)\right| & \leqq|f(z)-\Phi(\eta)|+|\Phi(\eta)-f(y)| \\
& +|f(y)-\Phi(\xi)|+\left|\Phi(\xi)-f\left(x_{i}\right)\right| \\
& <\frac{4}{K}=\frac{1}{4 n_{0}} .
\end{aligned}
$$

But this last inequality implies that $\omega(f \mid P, z) \leqq 1 / 2 n_{0}$, which is a contradiction.

It is of interest to note that the function $\Phi$ in Theorem 2 need not be continuous, and in fact, $\Phi$ need not even be measurable in the case where $X$ is $n$-dimensional euclidean space. For the case where $X=R^{2}$ (with the usual metric) we mention the following as a corollary.

COROLlaRY. Let $\Phi: R^{2} \times R^{+} \rightarrow R$ have the property that for each point $(x, y, 0)$ in the plane $z=0$ there is a cone in $R^{2} \times[0, \infty)$, with vertex at $(x, y, 0)$ and with its axis of symmetry perpendicular to the plane $z=0$, such that $\lim \Phi(u, v, w)$ exists as $(u, v, w)$ approaches $(x, y, 0)$ relative to the cone. If $f$ is the boundary function of $\Phi$ relative to these cones, then $f$ is in the first Baire class. (Observe that Stolz cones in $R^{2} \times R^{+}$are just ordinary cones.) 
It is also possible to modify Theorem 2 for a function $\Phi$ defined on a spherical ball in 3-space. That is, if $\Phi$ is any function defined on a ball in 3-space and if at each point of the boundary of the ball the limit of $\Phi$ exists relative to a cone whose axis of symmetry is a radius of the ball, then the boundary function thus obtained is in the first Baire class.

Concerning the corollary, it is natural to ask about the possibility of taking limits relative to cones which are "tilted." It is of course easy to see that this would result in non-Baire measurable functions. However, it is possible to allow some varying in the sets on which the limits are taken (see [5], Th. 2 and Th. 3), and in fact it is possible to allow the use of more general sets on which the limits are taken, that is, not just Stolz cones or generalized Stolz cones. This is the subject of the next theorem, but first it is necessary to recall some definitions.

Let $(X, \rho)$ be a bounded metric space and let $\mathscr{F}$ be the collection of all closed nonempty subsets of $X$. For $F \in \mathscr{F}$ and $\varepsilon$ positive, let

$$
V(F, \varepsilon)=\{x \in X: \rho(x, F)<\varepsilon\} .
$$

For $F, K \in \mathscr{F}$, set $h(F, K)=\inf \{\varepsilon: \varepsilon>0$,

$$
F \subset V(K, \varepsilon) \text { and } K \subset V(F, \varepsilon)\} .
$$

Then $h$ is the Hausdorff metric on $\mathscr{F}$ determined by $\rho$.

Let $(X, \rho)$ be a metric space, and

$$
\rho^{\prime}((x, r),(y, s))=\max \{\rho(x, y),|r-s|\}
$$

be the metric on $X \times[0, \infty)$. Then let $\infty$ be the collection of open sets $G$ in $X \times[0, \infty)$ such that $\partial G \cap(X \times\{0\})=\{(x, 0)\}$ for some $x \in X$, where $\partial G$ denotes the boundary of $G$. Then let $\overline{\mathcal{O}}$ be the collection of all Closure $(G), G \in \mathcal{O}$. With the induced Hausdorff metric $\odot$ becomes a metric space.

THeOREM 3. Let $X$ be a bounded $F_{I I}$ metric space and let $\overline{\mathcal{O}}$ be as above. Suppose that $G: X \rightarrow \overline{\mathcal{O}}$ is a continuous mapping such that $G(x) \cap(X \times\{0\})=\{(x, 0)\}$ for each $x \in X$, and suppose there is a function $\Phi: X \times R^{+} \rightarrow R$ such that for each $x \in X, \lim \Phi(u, r)$ exists as $(u, r)$ approaches $(x, 0)$ relative to the set $G(x)$. Then the boundary function $f$ of $\Phi$ for this collection of sets is in the first Baire class on $X$.

Proof. Let $P$ be a nonempty perfect subset and suppose $f \mid P$ has no point of continuity. Then let $D_{n}, D_{n_{0}} \sigma$, and $Q$ be as in the proof of Theorem 2. 
In the same fashion as before we let

$$
G(x)_{n}=\left\{(y, r) \in G(x): r \leqq \frac{1}{n}\right\}
$$

and set

$$
A_{n}=\left\{x \in Q: q \in G(x)_{n} \Rightarrow|f(x)-\Phi(q)|<\frac{1}{M}\right\}
$$

where $M=16 n_{0}$. It follows that $Q=\bigcup_{n=1}^{\infty} A_{n}$, and since $X$ is an $F_{I I}$ space, $Q$ is of the second category in itself. Thus there is an integer $k$ and an open sphere $\tau$ such that $Q \cap \tau$ is nonempty and $A_{k}$ is dense in $Q \cap \tau$.

Let $z$ be a fixed point in $A_{k} \cap \tau \cap \sigma$, and let $\zeta \in \operatorname{Int} .\left(G(z)_{k}\right)$. There is an open sphere in $X \times R^{+}$with center $\zeta$ and radius $\varepsilon$ which is contained in Int. $\left(G(Z)_{k}\right)$. Since $G: X \rightarrow \overline{\mathcal{O}}$ is continuous, there is an open sphere $V$ in $X$ with center $z$ such that $x \in V$ implies

$$
h(G(z), G(x))<\frac{\varepsilon}{2}
$$

(where $h$ denotes the Hausdorff metric). Consequently, $\rho^{\prime}(\zeta, G(x))<\varepsilon / 2$ for $x \in V$. Thus $\mathrm{G}(x)_{k} \cap G(z)_{k} \neq \varnothing$ for any $x \in V$.

The remainder of the proof is essentially the same as the latter part of the proof of Theorem 2 and so is omitted.

It would also be possible to use $\mathscr{O}$ in place of $\overline{\mathcal{O}}$ in Theorem 3 and require that $G: X \rightarrow \infty$ be continuous relative to the Hausdorff pseudo-metric on $\mathcal{O}$. We also remark that the sets $G(x)$ in Theorem 3 need not be connected, whereas in [5] the sets on which the limits were taken were always connected or had just two components. In his thesis the author gave an example of a function $\Phi: R \times R^{+} \rightarrow R$ such that the characteristic function of any subset of $R$ can be obtained as a boundary function of $\Phi$ by using open sets whose closures are connected as the sets on which the boundary limits are taken.

\section{References}

1. F. Hausdorff, Set Theory, (Transl. 3rd ed. of Mengenlehre, 1937), Chelsea, New York, 1957.

2. W. Hurewicz, Relativ perfekte Teile von Punktemengen und Mengen (A), Fund. Math. 12 (1928), 78-109.

3. V. Jarnik, Sur les Fonctions de la Première Classe de Baire, Bull. Internat. Acad. Sci. Boheme (1926).

4. G. Lederer, Two theorems on Baire functions in separable metric spaces, Quart. J. Math. Oxford Ser. 11 (1960), 269-274. 
5. L. Snyder, Continuous Stolz extensions and boundary functions, Trans. Amer. Math. Soc. 119 (1965), 417-427.

Received April 5, 1965. The results reported here are a portion of the author's doctoral dissertation written of Purdue University under the direction of Prof. C. J. Neugebauer. Supported in part by NSF Grant 18920.

UNIVERSITY OF VIRGINIA

Charlottesville, Virginia 


\section{PACIFIC JOURNAL OF MATHEMATICS}

\section{EDITORS}

\section{H. SAMELSON}

Stanford University

Stanford, California

J. P. JANS

University of Washington

Seattle, Washington 98105
J. DUGUNDJI

University of Southern California Los Angeles, California 90007

RICHARD ARENS

University of California

Los Angeles, California 90024

\section{ASSOCIATE EDITORS}
E. F. BECKENBACH
B. H. NEumanN
F. WOLF
K. YosidA

\section{SUPPORTING INSTITUTIONS}

\author{
UNIVERSITY OF BRITISH COLUMBIA \\ CALIFORNIA INSTITUTE OF TECHNOLOGY \\ UNIVERSITY OF CALIFORNIA \\ MONTANA STATE UNIVERSITY \\ UNIVERSITY OF NEVADA \\ NEW MEXICO STATE UNIVERSITY \\ OREGON STATE UNIVERSITY \\ UNIVERSITY OF OREGON \\ OSAKA UNIVERSITY \\ UNIVERSITY OF SOUTHERN CALIFORNIA
}

\author{
STANFORD UNIVERSITY \\ UNIVERSITY OF TOKYO \\ UNIVERSITY OF UTAH \\ WASHINGTON STATE UNIVERSITY \\ UNIVERSITY OF WASHINGTON \\ AMERICAN MATHEMATICAL SOCIETY \\ CHEVRON RESEARCH CORPORATION \\ TRW SYSTEMS \\ NAVAL ORDNANCE TEST STATION
}

Mathematical papers intended for publication in the Pacific Journal of Mathematics should be typewritten (double spaced). The first paragraph or two must be capable of being used separately as a synopsis of the entire paper. It should not contain references to the bibliography. Manuscripts may be sent to any one of the four editors. All other communications to the editors should be addressed to the managing editor, Richard Arens at the University of California, Los Angeles, California 90024.

50 reprints per author of each article are furnished free of charge; additional copies may be obtained at cost in multiples of 50 .

The Pacific Journal of Mathematics is published monthly. Effective with Volume 16 the price per volume ( 3 numbers) is $\$ 8.00$; single issues, $\$ 3.00$. Special price for current issues to individual faculty members of supporting institutions and to individual members of the American Mathematical Society: $\$ 4.00$ per volume; single issues $\$ 1.50$. Back numbers are available.

Subscriptions, orders for back numbers, and changes of address should be sent to Pacific Journal of Mathematics, 103 Highland Boulevard, Berkeley 8, California.

Printed at Kokusai Bunken Insatsusha (International Academic Printing Co., Ltd.), 7-17, Fujimi 2-chome, Chiyoda-ku, Tokyo, Japan.

PUBLISHED BY PACIFIC JOURNAL OF MATHEMATICS, A NON-PROFIT CORPORATION

The Supporting Institutions listed above contribute to the cost of publication of this Journal, but they are not owners or publishers and have no responsibility for its content or policies. 


\section{Pacific Journal of Mathematics

Vol. 22, No. $3 \quad$ March, 1967

Wai-Mee Ching and James Sai-Wing Wong, Multipliers and $H^{*}$

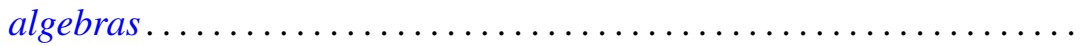

P. H. Doyle, III and John Gilbert Hocking, A generalization of the Wilder

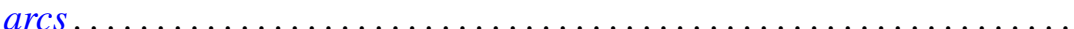

Irving Leonard Glicksberg, A Phragmén-Lindelöf theorem for function

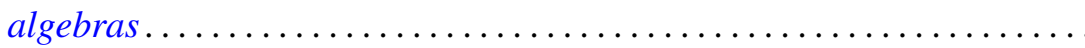

E. M. Horadam, A sum of a certain divisor function for arithmetical semi-groups ..................................... 407

V. Istrăţescu, On some hyponormal operators ................... 413

Harold H. Johnson, The non-invariance of hyperbolicity in partial

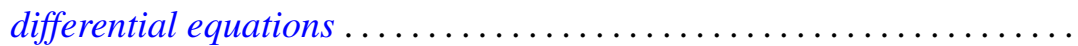

Daniel Paul Maki, On constructing distribution functions: A bounded denumerable spectrum with $n$ limit points................... 431

Ronald John Nunke, On the structure of Tor. II .................... 453

T. V. Panchapagesan, Unitary operators in Banach spaces ............. 465

Gerald H. Ryder, Boundary value problems for a class of nonlinear differential equations ................................. 477

Stephen Simons, The iterated limit condition and sequential convergence .................................... 505

Larry Eugene Snyder, Stolz angle convergence in metric spaces ......... 515 Sherman K. Stein, Factoring by subsets ................... 523

Ponnaluri Suryanarayana, The higher order differentiability of solutions of abstract evolution equations . . .

Leroy J. Warren and Henry Gilbert Bray, On the square-freeness of Fermat and Mersenne numbers ............................... 563

Tudor Zamfirescu, On l-simplicial convexity in vector spaces........... 565 Eduardo H. Zarantonello, The closure of the numerical range contains the spectrum 